non-fatal AMI within a population based case control study of urban Sri Lankans.

Methods Cases were patients consecutively admitted to the cardiology and general medical wards of the National Hospital of Sri Lanka with a confirmed diagnosis of AMI and discharged alive. Age and sex matched community controls were recruited within $20-30 \mathrm{~km}$ distance from National Hospital of Sri Lanka using the electoral lists. Basic demographic information, smoking habits, physical activity and dietary patterns, anthropometric indices, fasting glucose and serum lipid measurements were done on all participants.

Results During the study period, 262 cases and 246 controls were recruited. Of the non-fatal acute myocardial infarct patients, $18 \%$ were female. Compared to males, females with non-fatal events were significantly older (58 years, SD 6.6). Mean age among cases and controls were similar ( $\sim 54$ years). Cases were significantly more likely to have diabetes mellitus, a family history of AMI, abnormalities in lipid profile and report poor vegetable and fruit consumption (consumption was defined as "poor" if the individual did not usually consume some fruit/vegetable at least once on a given day). Physical activity patterns, education level and smoking habits were similar between cases and controls.

Conclusion Results indicate a high prevalence of modifiable risk factors among AMI patients. It is vital that the health system identify these patients early and provide them with optimal treatment.

\section{P2-248 TRENDS IN PROSTATE CANCER MORTALITY RATES IN THAILAND, 1998-2006}

doi:10.1136/jech.2011.142976j.81

${ }^{1}$ A Prasertchai, ${ }^{1}$ S Jitapunkul. 'School of Health Science, Sukhothai Thammathirat Open University, Nonthaburi, Thailand; ${ }^{2}$ Faculty of Medicine, Chulalongkorn University, Bangkok, Thailand

Introduction Prostate cancer is one of the leading cause of death among Thai males. In Thailand, whether mortality rates of prostate cancer have been increasing from1998 to 2006. This study aimed to examine trends in age-specific and age-standardised mortality rates for prostate cancer among Thai population.

Methods Mortality data during 1998-2006 were analysed based on ICD-10 for prostate cancer from Bureau of Health Policy and Planning, Ministry of Public Health. Both 5-year age-specific and agestandardised mortality rates per 100000 were calculated and descriptively analysed for trends.

Results The results have shown that during 1998-2006, agestandardised mortality rates for prostate cancer increased from 0.38 to 1.56 for males. For each year, prostate cancer mortality rates increased with age and peaked at age 70 and over. In 2006, the highest mortality rates were 45.76 for male.

Conclusion The prostate cancer mortality rates had increased at least fourfold in 8 years for both sexes. The increase in mortality might be explained by the increased in risk factors including increase in ageing of the population, eating habit characterised by high fat, obesity, and hormonal factors. Health policymakers should increase its effort in controlling and reducing the risk factors by promoting healthy behaviours such as healthy diet. It is also important to make an effort to inform the profile of prostate-specific antigen testing for screening prostate cancer.

\section{P2-249 SMOKELESS TOBACCO AND CORONARY HEART DISEASE IN BANGLADESH: IS THERE ANY ASSOCIATION?}

doi:10.1136/jech.2011.142976j.82

${ }^{1} \mathrm{M}$ A Rahman, ${ }^{1} \mathrm{~N}$ Spurrier, ${ }^{1} \mathrm{M}$ A Mahmood, ${ }^{2} \mathrm{M}$ Rahman, ${ }^{3} \mathrm{~S} \mathrm{R}$ Choudhury, ${ }^{4} \mathrm{~S}$ Leeder. ${ }^{1}$ Discipline of Public Health, The University of Adelaide, Adelaide, South
Australia, Australia; ${ }^{2}$ IEDCR (Institute of Epidemiology, Disease Control and Research), Dhaka, Bangladesh; ${ }^{3}$ NHFH\&RI (National Heart Foundation Hospital \& Research Institute), Dhaka, Bangladesh; ${ }^{4}$ The Menzies Centre for Health Policy, The University of Sydney, Sydney, New South Wales, Australia

Objective To determine the association between smokeless tobacco (SLT) use and coronary heart disease (CHD) among non-smoking adults in Bangladesh.

Methods A case-control study of non-smoking Bangladeshi adults aged $40-75$ years, was conducted in 2010. Incident cases of CHD were selected from two cardiac hospitals. Hospital controls were selected from outpatient departments of the same hospitals. Community controls were selected from neighbourhoods matched to CHD cases. Four community controls and one hospital control were matched to each case on age and gender.

Results The study enrolled 302 cases, 1208 community controls and 302 hospital controls. Forty percent of the study subjects were current users of or had used SLT in the past. Current use of SLT was similar among cases (33\%), community controls (33\%) and hospital controls (32\%). Current use of SLT was not associated with increased risk of $\mathrm{CHD}$ when community controls were used (adjusted OR $0.87,95 \%$ CI 0.63 to $1.19, \mathrm{p}>0.05$ ), or hospital controls were used (adjusted OR 1.00, 95\% CI 0.63 to 1.60, p>0.05), or when both controls were combined (adjusted OR 1.00, 95\% CI 0.74 to $1.34, p>0.05$ ). Risk of CHD did not increase with use of individual type, frequency, duration and past use of SLT products.

Conclusion In this study, there was no statistically significant association between SLT use and CHD among non-smoking adults in Bangladesh. If the findings can be replicated in prospective studies, it may well be that strategic focus for reducing CHD in Bangladesh should be upon smoking control rather than on SLT.

\section{P2-250 OMEGA 3 POLYUNSATURATED FATTY ACIDS (PUFAS) AND RISK OF EARLY ONSET PROSTATE CANCER}

doi:10.1136/jech.2011.142976j.83

${ }^{1}$ A Atta Ur Rahman, ${ }^{*}$ A Lophatananon, ${ }^{3} \mathrm{~J}$ Lobaz, ${ }^{4} \mathrm{~F}$ Robinson, ${ }^{2} \mathrm{~S} S \mathrm{~S}$ Brown, ${ }^{4} \mathrm{~T}$ Parker, ${ }^{5}$ D Easton, ${ }^{6} \mathrm{Z}$ Kote-Jarai, ${ }^{7} \mathrm{R}$ Pocock, ${ }^{6} \mathrm{D}$ Dearnaley, ${ }^{6} \mathrm{M}$ Guy, ${ }^{6} \mathrm{R}$ A Wilkinson, ${ }^{9} \mathrm{~A}$ L Hall, ${ }^{6}$ E Sawyer, ${ }^{6} \mathrm{E}$ Page, ${ }^{8} \mathrm{~J}-\mathrm{F}$ Liu, ${ }^{6,9} \mathrm{R}$ A Eeles, ${ }^{2} \mathrm{~K}$ R Muir. ${ }^{1}$ Community Medicine and Public Health Sciences, Liaquat University of Medical and Health Sciences, Jamshoro, Sindh, Pakistan; ${ }^{2}$ Health Sciences Research Institute, Warwick Medical School, Warwick University, Coventry, UK; ${ }^{3}$ Division of Epidemiology and Public Health, University of Nottingham, Nottingham, UK; ${ }^{4}$ School of Biomedical sciences, University of Nottingham, Nottingham, UK; ${ }^{5}$ CR-UK Genetic Epidemiology Unit, Strangeways Research Laboratories, Worts Causeway, Cambridge, UK; ${ }^{6}$ Institute of Cancer Research, Sutton, Surrey, UK; ${ }^{7}$ Royal Devon and Exeter NHS Foundation Trust, Barrack Road, Exeter, UK; ${ }^{8}$ Children's Brain Tumour Research Centre, Division of Human Development, University of Nottingham, Queens Medical Centre, Nottingham, UK; ${ }^{9}$ The Royal Marsden NHS Foundation Trust, Downs Road, Sutton, UK

Introduction In the UK, approximately $11 \%$ of newly registered $\mathrm{PrCa}$ cases are under the age of 60 years. The unequal incidence across different countries suggests that modifiable factors, such as dietary intake of omega 3 polyunsaturated fatty acids (PUFAs) may be important.

Methods Data were analysed on 805 cases and 1283 controls of age $<60$ years. A food frequency questionnaire assessing typical diet 5 years previous to either diagnosis in the cases or returning questionnaire in controls was used to assess dietary PUFAs. Nutrient intake of specific PUFA derivatives was then calculated via a nutritional database. Unconditional logistic regression was used to calculate ORs and 95\% CIs for the effect of omega 3 and its derivatives docosahexaenoic acid (DHA), eicosapentaenoic acid (EPA), $\alpha$ linolenic acid ( $\alpha \mathrm{LNA}$ ) and supplementation of total omega 3, DHA and EPA on PrCa risk after adjusting for confounders. Linear trend was also assessed. 